\title{
Role of Thalamocortical Sensory Suppression during Arousal: Focusing Sensory Inputs in Neocortex
}

\author{
Manuel A. Castro-Alamancos \\ Department of Neurology and Neurosurgery, Montreal Neurological Institute, McGill University, Montreal, \\ Quebec H3A 2B4, Canada
}

The thalamus serves as a gate that regulates the flow of sensory inputs to the neocortex, and this gate is controlled by neuromodulators from the brainstem reticular formation that are released during arousal. We found recently that sensoryevoked responses are suppressed in the neocortex during arousal. This sensory suppression results from the activitydependent depression of the thalamocortical connection caused by increased tonic firing of thalamocortical cells during arousal. In the present study, the functional consequences of thalamocortical suppression during arousal were investigated using the vibrissae system of rodents. The results show that thalamocortical suppression is associated with a strong reduction in the spread of sensory inputs through the cortex, thus

During arousal states typical of information processing, electroencephalographic activity is characterized by low-amplitude fast activity, called activation, which contrasts with the largeamplitude regular and slow activity typical of quiescent states (Moruzzi and Magoun, 1949). Activation is produced by the release of several neuromodulators in the thalamus and cortex. For instance, during arousal, cells in the brainstem laterodorsal tegmentum increase their firing rates (Steriade et al., 1990), releasing acetylcholine in the thalamus (Williams et al., 1994), which depolarizes thalamocortical neurons (McCormick, 1992), increasing their spontaneous firing rates in the tonic firing mode and enhancing the transmission of low-frequency sensory inputs through the thalamus (Steriade, 1969; Singer, 1977; Steriade et al., 1997; Sherman and Guillery, 2001). The postsynaptic depolarization of thalamocortical neurons during arousal also strongly facilitates the transmission of high-frequency sensory inputs through the thalamus (Castro-Alamancos, 2002a,b), which are normally filtered during quiescent states. In addition, the increased firing rate of thalamocortical neurons during arousal leads to the activity-dependent depression of thalamocortical synapses producing thalamocortical sensory suppression (CastroAlamancos and Oldford, 2002). Thus, during aroused states,

\footnotetext{
Received July 29, 2002; revised Aug. 23, 2002; accepted Sept. 3, 2002.

This work was supported by the Medical Research Council of Canada, Natural Sciences and Engineering Council of Canada, Fonds de la Recherche en Santé du Quebec, Canadian Foundation for Innovation, and Savoy Foundation. Multichannel silicon probes were provided by the University of Michigan Center for Neural Communication Technology sponsored by National Institutes of Health National Center for Research Resources. Thanks to Dan Simons and Harvey Swadlow for helpful comments.

Correspondence should be addressed to Dr. Manuel Castro-Alamancos, Montreal Neurological Institute, 3801 University Street, Room WB210, Montreal, Quebec H3A 2B4 Canada. E-mail: manuel.castro@mcgill.ca.

Copyright (C) 2002 Society for Neuroscience 0270-6474/02/229651-05\$15.00/0
}

reducing the size of sensory representations. In addition, when the responses of single cells to principal and adjacent whiskers are compared, the response to the adjacent whiskers was found to be strongly suppressed, much more so than that of principal whiskers. Consequently, the receptive fields of cortical neurons become more focused to the principal whisker. The results indicate that thalamocortical suppression during arousal serves to focus sensory inputs to their appropriate representations in neocortex, which may be computationally helpful for the spatial processing of sensory information.

Key words: receptive field; whisker; barrel cortex; synaptic depression; sensory processing; behavioral state there is an increase in the efficacy of sensory transmission through the thalamus, especially for high-frequency inputs and, because of the increased firing of thalamocortical cells sensory inputs reaching the neocortex during arousal, encounter a depressed thalamocortical synapse, which causes cortical sensory suppression. The aim of the present study is to investigate some of the functional consequences of cortical sensory suppression during arousal.

During anesthesia or quiescent states, sensory inputs spread through large areas of neocortex, giving rise to large receptive fields and sensory representations (Armstrong-James and Fox, 1987; Armstrong-James et al., 1991; Chen-Bee and Frostig, 1996; Moore and Nelson, 1998; Sheth et al., 1998; Ghazanfar et al., 2000; Petersen and Diamond, 2000; Brett-Green et al., 2001). These observations suggest that, during quiescent states, the neocortex favors the spread of activity. Other studies also in the barrel cortex of rodents have found instead a great spatial contrast between adjacent whiskers in the barrel cortex (Simons and Carvell, 1989; Goldreich et al., 1999), primarily mediated by locally recurrent inhibition (Simons, 1995). The more restricted receptive fields and barrel independence found by these studies has been attributed to the use of different anesthetics (Simons et al., 1992), which produce different levels of brain activation. Thus, receptive fields and cortical representations may differ between different levels of arousal. The present study investigated the effect of brain activation caused by stimulating the brainstem reticular formation on the responses of cortical neurons to whisker stimulation. The hypothesis is that thalamocortical sensory suppression during arousal serves to focus sensory inputs in neocortex (Castro-Alamancos and Oldford, 2002). Sensory representations and receptive fields in the barrel cortex of rodents were compared in anesthetized rats between quiescent states and 
A

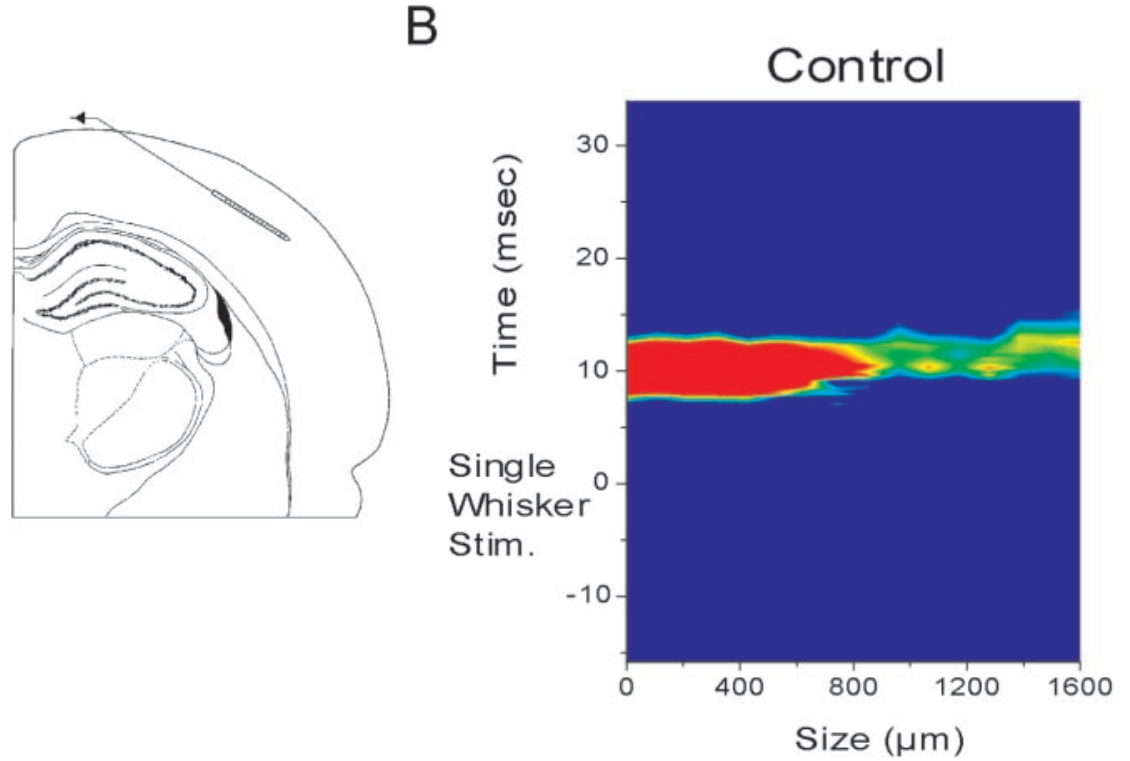

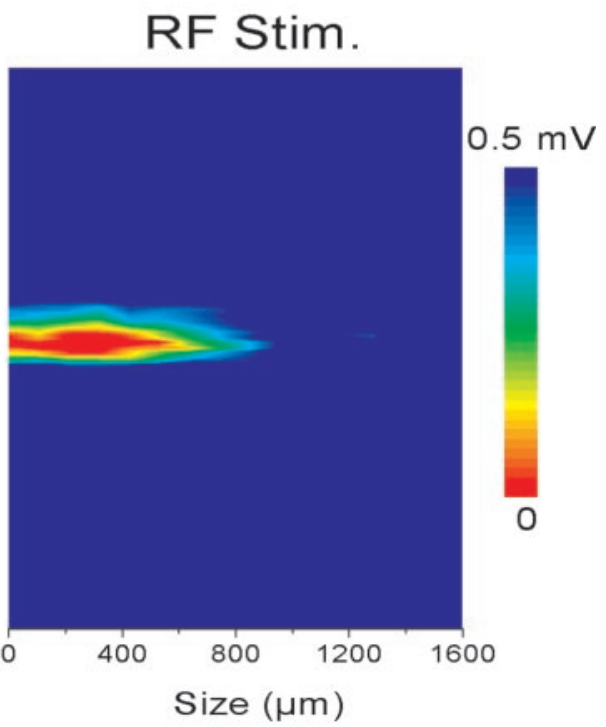

Figure 1. Sensory representations in neocortex are focused during activation. $A$, Schematic representation of the location of the 16 channel silicon probe placed at a $135^{\circ}$ angle in the barrel cortex to record field potential responses through an extension of layers IV-III of the barrel neocortex. $B$, Contour plot of the amplitude of the negative field potential recorded from 16 sites (100 $\mu \mathrm{m}$ intervals) along layers IV-III of the barrel cortex in response to stimulation of a single whisker. Note the spread of activity under control conditions and the suppression of spread and focusing of the representation after brainstem reticular formation stimulation (RF Stim.).

activated states produced by stimulating the brainstem reticular formation.

\section{MATERIALS AND METHODS}

Surgical procedures. Adult Sprague Dawley rats (300 gm) were anesthetized with urethane $(1.5 \mathrm{gm} / \mathrm{kg}$, i.p. $)$ and placed in a stereotaxic frame. All skin incisions and frame contacts with the skin were injected with lidocaine (2\%). A unilateral craniotomy extended over a large area of the parietal cortex. Small incisions were made in the dura as necessary, and the cortical surface was covered with artificial CSF (ACSF) containing the following (in mM): $126 \mathrm{NaCl}, 3 \mathrm{KCl}, 1.25 \mathrm{NaH}_{2} \mathrm{PO} 4,26 \mathrm{NaHCO}_{3}, 1.3$ $\mathrm{MgSO}_{4} 7 \mathrm{H}_{2} \mathrm{O}, 10$ dextrose, and $2.5 \mathrm{CaCl}_{2} 2 \mathrm{H}_{2} \mathrm{O}$. Body temperature was automatically maintained constant with a heating pad. The level of anesthesia was monitored with field recordings and limb-withdrawal reflexes and kept constant at approximately stage III/3 using supplemental doses of urethane (Friedberg et al., 1999). At the end of the experiments, the animals were killed with an overdose of sodium pentobarbitone (intraperitoneally). The Animal Care Committee of McGill University approved protocols for all experiments.

Electrophysiological procedures. Extracellular recordings were performed using electrodes (5-10 M $\Omega$ ) filled with ACSF; single units and field potentials were recorded simultaneously via the same electrodes located in the primary somatosensory neocortex (barrel cortex) at a depth between 400 and $900 \mu \mathrm{m}$ (i.e., layers IV-III). These highimpedance electrodes recorded an isolated single neuron. The exact location of the recorded cells was not determined using histological procedures. Coordinates (Paxinos and Watson, 1982) for the stimulating electrode in the laterodorsal tegmentum (brainstem reticular formation; $100 \mathrm{~Hz}, 1-\mathrm{sec}$ ) were as follows (in $\mathrm{mm}$, from bregma and the dura): posterior, 9; lateral, 0.7; depth, 5-6. Electrical stimuli consisted of 200 $\mu$ sec pulses of $<200 \mu \mathrm{A}$ and were evoked using a concentric stimulating electrode (200- $\mu \mathrm{m}$-diameter ultra-small concentric bipolar electrode; Frederick Haer Co., Bowdoinham, ME).

Sensory stimulation. The sensory stimulation consisted of deflecting single whiskers. Two stimulators were used that allowed independently stimulating two whiskers: the principal whisker and an adjacent whisker. The principal whisker was defined as the one producing the strongest response for the recorded single neuron. This was determined by using a hand-held probe to map the individual responses. The adjacent whisker was one that also discharged the recorded neuron. The selected whiskers were inserted into stimulators consisting of glass micropipettes (1-mmdiameter) glued to the membrane of a miniature speaker. During the experiment, stimulation to each whisker alternated at 4-12 sec intervals. Application of a $1 \mathrm{msec}$ square current pulse to the speaker deflected the micropipette and the whisker inside $\sim 400 \mu \mathrm{m}$. The whisker-evoked response was measured between 5 and $15 \mathrm{msec}$ after the whisker stimulus. Whisker stimulation was applied between 0.5 and $10 \mathrm{sec}$ after the brainstem reticular formation stimulation.

Analysis of horizontal spread. A 16 channel linear silicon probe with 100 $\mu \mathrm{m}$ site spacing $(\sim 50-\mu \mathrm{m}$-diameter; Center for Neural Communication Technology, University of Michigan, Ann Arbor, MI) was placed in layers IV-III of the barrel cortex parallel to the pial surface. This required inserting the silicon probe with a $315^{\circ}$ angle (in the coronal plane) at $3.5 \mathrm{~mm}$ lateral from the midline. The probe was advanced $\sim 3$ $\mathrm{mm}$ from the pia and thus placed horizontally in layers IV-III. In several animals $(n=2)$, the location of the probe along layers IV-III was confirmed by preparing coronal slices in ACSF using standard brain slicing procedures (Castro-Alamancos, 2002b). This allowed visualizing in a microscope the tract of the probe penetration. In both cases, the recording sites of the probe were estimated to be between 650 and 850 $\mu \mathrm{m}$ from the surface. No histological procedure was used. Field potential recordings were obtained simultaneously from the 16 sites on the probe in response to deflections of a single whisker. To equalize the impedance in each site on the probe $(\sim 500 \mathrm{~K} \Omega)$, they were oxidized before use (Castro-Alamancos, 2000).

\section{RESULTS}

Thalamocortical suppression during activated states may serve to focus sensory inputs to their specific cortical representations by limiting the spread of activity. This was tested by monitoring the cortical responses along a horizontal strip $(1.6 \mathrm{~mm})$ of the barrel cortex along layers IV-III with $100 \mu \mathrm{m}$ resolution using a 16 channel silicon probe (Fig. $1 A$ ). Examples of the field potential responses recorded with this method have been published previously (Castro-Alamancos and Oldford, 2002). Deflecting a single whisker produces responses in a large portion of barrel cortex that exceeds the area of a single cortical barrel (Petersen and Diamond, 2000; Brett-Green et al., 2001). In fact, the extent of the cortex responding to stimulation of a single whisker was larger than the extent of the $1.6 \mathrm{~mm}$ probe. The activity in neocortex 
spreads horizontally through barrel cortex as reflected by the longer latencies of the responses recorded surrounding the shortlatency and large-amplitude response in the center (Fig. 1B). However, activation induced by brainstem reticular formation stimulation strongly limited the size of the neocortical area responding to a single whisker (Fig. 1B). During activation, stimulation of a single whisker produced a response in the neocortex that showed little spread to adjacent territory. As a consequence, during activated states, the cortical response to stimulation of single whiskers was focused to a significantly smaller area. Based on several rats $(n=4)$, the relative spread of activity from the center of the representation (i.e., the recording site on the probe with the shortest latency response; $\sim 5.5 \mathrm{msec}$ ) was reduced by $59.5 \pm 3 \%(p>0.0001 ; t$ test $)$ during activation caused by stimulating the brainstem reticular formation. This relative measure underestimates the amount of reduction in spread caused by activation because the complete extent of the spread was usually larger than the probe.

Because the spread of activity through the cortex is focused considerably during activation, this should also be manifested in the focusing of the receptive fields in cortical cells from layers IV-III. To test this hypothesis, single-unit recordings were obtained from cells in layers IV-III of the barrel cortex $(n=23$ cells). No effort was made to distinguish between these two layers in the present study. Stimulation of the brainstem reticular formation resulted in either an enhancement or reduction of the spontaneous firing rate of the cortical neurons recorded, but, as shown previously, they all reduced their response to the sensory stimulus (Castro-Alamancos and Oldford, 2002). The mean spontaneous firing rate of all of the recorded cells was $3.2 \pm 0.5 \mathrm{~Hz}$ during control conditions and $6.1 \pm 1 \mathrm{~Hz}$ during activation. From this population of cells, $34 \%(n=8$ of 23$)$ enhanced their firing rate, whereas $66 \%(n=16$ of 23$)$ reduced their firing rate during activation compared with control. The cells that enhanced their firing rate $(n=8)$ had a spontaneous firing rate of $5.6 \pm 2 \mathrm{~Hz}$ during control conditions and $16.2 \pm 3 \mathrm{~Hz}$ during activation ( $t$ test; $p<0.001)$, whereas those remaining $(n=15)$ had a firing rate of $2.1 \pm 1 \mathrm{~Hz}$ during control and $0.6 \pm 0.2 \mathrm{~Hz}$ during activation $(t$ test; $p<0.01)$.

After determining the principal whisker corresponding to the recorded neuron and an adjacent whisker that also discharged the neuron, a baseline was obtained under control conditions $(n=$ 60-100 trials per whisker). This yielded a large short-latency response to the principal whisker and a smaller and longerlatency response to the adjacent whisker. Figure 2 presents an example of a cell recorded at $720 \mu \mathrm{m}$ in depth in the barrel cortex. The cell responded robustly to the principal whisker and also to an adjacent whisker (Fig. $2 A$ ). Stimulation of the brainstem reticular formation reduced the spontaneous firing rate of this neuron and also reduced the sensory responses to the principal and the adjacent whiskers. However, whereas the response to the adjacent whisker was virtually eliminated, the response to the principal whisker was still salient. Consequently, a strong selectivity of the response for the principal whisker resulted, i.e., during aroused states of the brain, cortical neurons become more selective for their principal inputs. Among the recorded neurons, several $(n=5)$ displayed features that are consistent with inhibitory interneurons (Swadlow, 1995). These suspected interneurons responded with a high-frequency burst to whisker stimulation and displayed short spike durations. Interestingly, the sensory response of these neurons was also focused by activation. Figure 3 presents an example of a response from a presumed

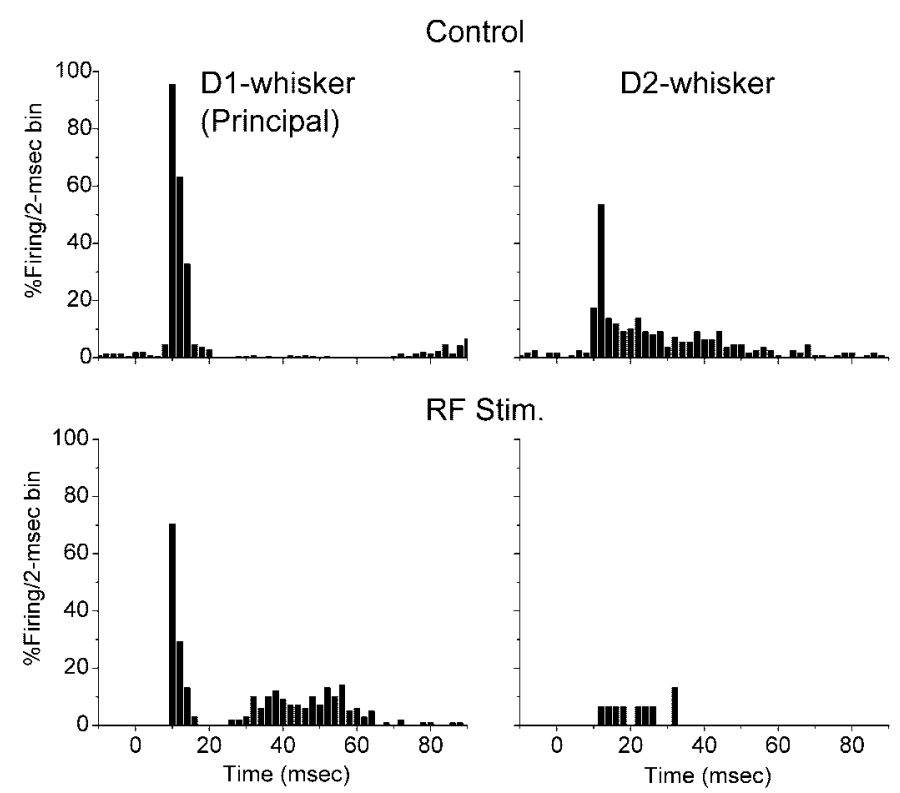

Figure 2. Enhanced selectivity of a neuron in layers IV-III of barrel cortex during activation. Effect of activation induced by stimulating the brainstem reticular formation on single-unit response to stimulation of the principal whisker and of an adjacent whisker. Single-unit responses are displayed as the probability of firing per $2 \mathrm{msec}$ bins before (top) and during (bottom) activation (RF Stim.). The whisker stimulus is delivered at time 0 .

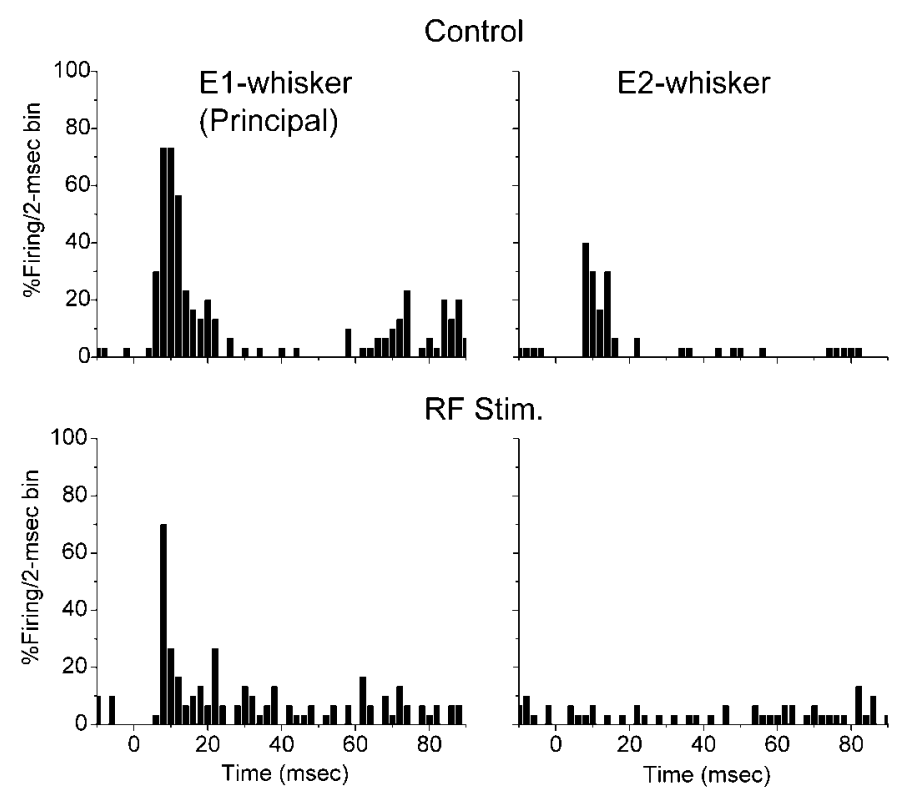

Figure 3. Enhanced selectivity of a presumed inhibitory interneuron in layers IV-III of barrel cortex during activation. Effect of activation induced by stimulating the brainstem reticular formation on single-unit response to stimulation of the principal whisker (left) and of an adjacent whisker (right). Single-unit responses are displayed as the probability of firing per 2 msec bins before (top) and during (bottom) activation (RF Stim.). The whisker stimulus is delivered at time 0.

interneuron located at $690 \mu \mathrm{m}$ in depth. The cell responded robustly to the principal whisker and also to the adjacent whisker during quiescent states. After activation induced by stimulating the brainstem reticular formation, this neuron enhanced its spontaneous firing rate, whereas the response to both the principal and 

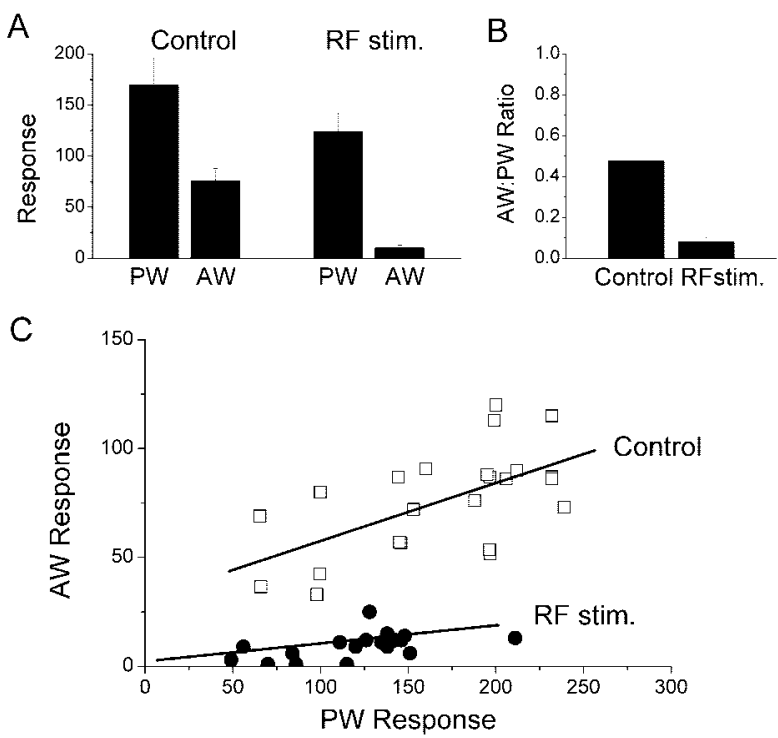

Figure 4. Population data showing the enhanced selectivity of neurons in layers IV-III of barrel cortex during activation. $A$, Mean effect of activation induced by stimulating the brainstem reticular formation on the response of single units $(n=23)$ to stimulation of the principal whisker $(P W)$ and of an adjacent whisker $(A W)$. $B$, Ratio of the adjacent whisker and principal whisker responses during control conditions and during activation caused by stimulating the brainstem reticular formation $(R F$ Stim.). $C$, Scatterplot for each single unit displaying the principal whisker response plotted versus the adjacent whisker response under control conditions (open squares) and during activation caused by stimulating the brainstem reticular formation ( filled circles). A regression line is used to fit each group of data.

adjacent whiskers were suppressed. However, the response suppression resulted in the complete elimination of the response to the adjacent whisker, whereas the response to the principal whisker was still present. Thus, presumed interneurons also enhance their selectivity during arousal as a consequence of sensory suppression.

Figure 4 presents population data for the cells in the study $(n=$ $23)$. For the cells tested in layers IV-III, the response to the principal whisker was reduced by $26.6 \pm 3 \%$ ( $t$ test; $p<0.001)$, whereas the response to the adjacent whisker was reduced by $87.5 \pm 2 \%(t$ test; $p<0.0001)$ (Fig. $4 A)$. As a consequence of the much stronger reduction in the response to the adjacent whisker, the response of the neurons became selective for the principal whisker. This selectivity was further assessed by calculating a ratio $(A D / P W)$ between the response to the adjacent whisker and the principal whisker (Fig. $4 B$ ). Under control conditions, the mean ratio was $0.47 \pm 0.08$. However, during activation caused by stimulating the brainstem reticular formation, the ratio was reduced to $0.08 \pm 0.02$, indicating that the preference for the principal whisker increased significantly ( $t$ test; $p<0.0001)$. Figure $4 C$ displays a scatterplot for the principal whisker response for each neuron plotted versus its adjacent whisker response under control conditions (open squares) and during activation caused by stimulating the brainstem reticular formation (filled circles). A regression line is used to fit each group of data. The responses to both adjacent and principal whiskers were reduced during activation. However, the response to the adjacent whisker was almost eliminated for most of the cells recorded. Thus, the cells became selective for the principal whisker.

\section{DISCUSSION}

The present study found that, because of sensory suppression, the horizontal spread of activity in the neocortex from the thalamocortical recipient area is strongly suppressed during activated states, resulting in the focusing of cortical sensory representations and receptive fields. Thus, during aroused states, thalamocortical suppression serves as a mechanism to focus sensory inputs to their appropriate representations (barrels) in neocortex, which may be helpful for the spatial processing of sensory information.

This focusing may be helpful for sensory processing because the lack of selectivity observed during quiescent states seems to hinder information processing. For example, simple tasks performed by the somatosensory system (e.g., two-point discrimination) are more difficult to conceive with such overlapping and large cortical representations and receptive fields. Obviously, these behavioral capacities are possible only during brainactivated states typical of alertness, attention, and arousal and not during quiescent states typical of drowsiness, inattentiveness, and sleep. Thus, to understand how these behavioral capacities are executed, it is important to study the properties of neural circuits during brain-activated states typical of arousal and information processing. Cortical sensory suppression during arousal may serve to focus sensory inputs to allow a more discreet and segregate representation of sensory information in the neocortex.

The activity-dependent suppression of the thalamocortical connection during arousal may work together with recurrent cortical inhibition to produce the effects described in the present study. Locally recurrent inhibition has been proposed as the means to achieve selectivity in the neocortex (Simons, 1985; Miller et al., 2001). In fact, thalamocortical neurons produce a very powerful connection with cortical inhibitory interneurons (Swadlow, 1995), much more so than with excitatory neurons of layer IV (Gibson et al., 1999). Although the efficacy of the thalamocortical connection with interneurons is reduced during aroused states (Swadlow and Gusev, 2001; Castro-Alamancos and Oldford, 2002), it should still be effective in producing inhibitory potentials and reducing the spread of activity. In fact, one of the main consequences of stimulating the brainstem reticular formation is a reduction of the spontaneous firing rate of a large percentage of cortical neurons (Castro-Alamancos and Oldford, 2002). This is consistent with the hyperpolarization of cortical neurons via the activation of $\mathrm{GABA}_{\mathrm{A}}$ receptors. One possibility is that the enhanced firing rates of thalamocortical neurons during arousal increase the firing of cortical inhibitory interneurons that are strongly innervated by thalamocortical inputs (White and Rock, 1981). This would result in an enhanced tonic level of recurrent inhibition in the neocortex during activated states. Therefore, thalamocortical suppression and enhanced cortical inhibition during arousal, which are both a direct consequence of enhanced thalamocortical firing, result in the increased selectivity (focusing) of cortical receptive fields and sensory representations.

Previous work has shown that, when receptive fields obtained during anesthesia are compared with those of waking animals, important transformations are observed. Some studies have shown that the size of cortical receptive fields increase as the depth of anesthesia is lessened from very deep levels (ArmstrongJames and George, 1988). Furthermore, when urethane anesthesia was compared with waking, neurons in the thalamocortical recipient layer (i.e., layer IV) had smaller receptive fields during waking compared with anesthesia, as indicated by multiunit recordings and comparing different cells in both states (Simons et 
al., 1992). In the visual system, cortical neurons also seem to reduce the size of their receptive fields during arousal (Worgotter et al., 1998). In the auditory system, different neurons undergo a variety of changes in receptive field size (Edeline et al., 2001). Also, in the auditory cortex, neurons undergo dramatic changes in receptive fields, so that after behavioral conditioning receptive fields enhance their response to the behaviorally relevant stimulus and reduce their response to other irrelevant stimuli (Weinberger, 1995). Although the present study was performed in urethane-anesthetized animals, it is important to point out that the thalamocortical responsiveness observed here during control versus activation states highly resembles the sleep-quiescent versus active exploration states in freely behaving animals (CastroAlamancos and Oldford, 2002). Thus, thalamocortical suppression is present in freely behaving animals during activated states, an effect that is mimicked in anesthetized animals by stimulating the brainstem reticular formation.

In conclusion, increased thalamocortical tonic firing during activation reduces the strength of the thalamocortical connection and may increase tonic cortical inhibition. Under these conditions, the response of cortical neurons to sensory inputs becomes more selective for their principal input. Thus, cortical representations and receptive fields become focused during arousal.

\section{REFERENCES}

Armstrong-James M, Fox K (1987) Spatiotemporal convergence and divergence in the rat S1 "barrel" cortex. J Comp Neurol 263:265-281.

Armstrong-James M, George MJ (1988) Influence of anesthesia on spontaneous activity and receptive field size of single units in rat Sm1 neocortex. Exp Neurol 99:369-387.

Armstrong-James M, Callahan CA, Friedman MA (1991) Thalamocortical processing of vibrissal information in the rat. I. Intracortical origins of surround but not centre-receptive fields of layer IV neurones in the rat S1 barrel field cortex. J Comp Neurol 303:193-210.

Brett-Green BA, Chen-Bee CH, Frostig RD (2001) Comparing the functional representations of central and border whiskers in rat primary somatosensory cortex. J Neurosci 21:9944-9954.

Castro-Alamancos MA (2000) Origin of synchronized oscillations induced by neocortical disinhibition in vivo. J Neurosci 20:9195-9206.

Castro-Alamancos MA (2002a) Different temporal processing of sensory inputs in the rat thalamus during quiescent and information processing states in vivo. J Physiol (Lond) 539:567-578.

Castro-Alamancos MA (2002b) Properties of primary sensory (lemniscal) synapses in the ventrobasal thalamus and the relay of highfrequency sensory inputs. J Neurophysiol 87:946-953.

Castro-Alamancos MA, Oldford E (2002) Cortical sensory suppression during arousal is due to the activity-dependent depression of thalamocortical synapses. J Physiol (Lond) 541:319-331.

Chen-Bee CH, Frostig RD (1996) Variability and interhemispheric asymmetry of single-whisker functional representations in rat barrel cortex. J Neurophysiol 76:884-894.

Edeline JM, Dutrieux G, Manunta Y, Hennevin E (2001) Diversity of receptive field changes in auditory cortex during natural sleep. Eur J Neurosci 14:1865-1880.

Friedberg MH, Lee SM, Ebner FF (1999) Modulation of receptive field properties of thalamic somatosensory neurons by the depth of anesthesia. J Neurophysiol 81:2243-2252.

Ghazanfar AA, Stambaugh CR, Nicolelis MA (2000) Encoding of tac- tile stimulus location by somatosensory thalamocortical ensembles. J Neurosci 20:3761-3775.

Gibson JR, Beierlein M, Connors BW (1999) Two networks of electrically coupled inhibitory neurons in neocortex. Nature 402:75-79.

Goldreich D, Kyriazi HT, Simons DJ (1999) Functional independence of layer IV barrels in rodent somatosensory cortex. J Neurophysiol $82: 1311-1316$.

McCormick DA (1992) Neurotransmitter actions in the thalamus and cerebral cortex and their role in neuromodulation of thalamocortical activity. Prog Neurobiol 39:337-388.

Miller KD, Pinto DJ, Simons DJ (2001) Processing in layer 4 of the neocortical circuit: new insights from visual and somatosensory cortex. Curr Opin Neurobiol 11:488-497.

Moore CI, Nelson SB (1998) Spatio-temporal subthreshold receptive fields in the vibrissa representation of rat primary somatosensory cortex. J Neurophysiol 80:2882-2892.

Moruzzi G, Magoun HW (1949) Brain stem reticular formation and activation of the EEG. Electroencephalogr Clin Neurophysiol 1:455-473.

Paxinos G, Watson C (1982) The rat brain in stereotaxic coordinates. New York: Academic.

Petersen RS, Diamond ME (2000) Spatial-temporal distribution of whisker-evoked activity in rat somatosensory cortex and the coding of stimulus location. J Neurosci 20:6135-6143.

Sherman SM, Guillery RW (2001) Exploring the thalamus. San Diego: Academic.

Sheth BR, Moore CI, Sur M (1998) Temporal modulation of spatial borders in rat barrel cortex. J Neurophysiol 79:464-470.

Simons DJ (1985) Temporal and spatial integration in the rat SI vibrissa cortex. J Neurophysiol 54:615-635.

Simons DJ (1995) Neuronal integration in the somatosensory whisker/ barrel cortex. In: Cerebral cortex, Vol 11, The barrel cortex of rodents (Jones EG, Diamond IT, eds), pp 263-298. New York: Plenum.

Simons DJ, Carvell GE (1989) Thalamocortical response transformation in the rat vibrissa/barrel system. J Neurophysiol 61:311-330.

Simons DJ, Carvell GE, Hershey AE, Bryant DP (1992) Responses of barrel cortex neurons in awake rats and effects of urethane anesthesia. Exp Brain Res 91:259-272.

Singer W (1977) Control of thalamic transmission by corticofugal and ascending reticular pathways in the visual system. Physiol Rev $57: 386-420$.

Steriade M (1969) Alteration of motor and somesthetic thalamo-cortical responsiveness during wakefulness and sleep. Electroencephalogr Clin Neurophysiol 26:334.

Steriade M, Datta S, Pare D, Oakson G, Curro Dossi RC (1990) Neuronal activities in brain-stem cholinergic nuclei related to tonic activation processes in thalamocortical systems. J Neurosci 10:2541-2559.

Steriade M, Jones EG, McCormick DA (1997) Thalamus. New York: Elsevier.

Swadlow HA (1995) Influence of VPM afferents on putative inhibitory interneurons in $\mathrm{S} 1$ of the awake rabbit: evidence from cross-correlation, microstimulation, and latencies to peripheral sensory stimulation. J Neurophysiol 73:1584-1599.

Swadlow HA, Gusev AG (2001) The impact of "bursting" thalamic impulses at a neocortical synapse. Nat Neurosci 4:402-408.

Weinberger NM (1995) Dynamic regulation of receptive fields and maps in the adult sensory cortex. Annu Rev Neurosci 18:129-158.

White EL, Rock MP (1981) A comparison of thalamocortical and other synaptic inputs to dendrites of two non-spiny neurons in a single barrel of mouse SmI cortex. J Comp Neurol 195:265-277.

Williams JA, Comisarow J, Day J, Fibiger HC, Reiner PB (1994) Statedependent release of acetylcholine in rat thalamus measured by in vivo microdialysis. J Neurosci 14:5236-5242.

Worgotter F, Suder K, Zhao Y, Kerscher N, Eysel UT, Funke K (1998) State-dependent receptive-field restructuring in the visual cortex. Nature 396:165-168. 